\title{
Camera Calibration with Accuracy Evaluation and Real-time Evaluation in the Use of Vision Robot
}

\author{
Zhen Fan ${ }^{1, a}$, Lei Zhang ${ }^{2, b}$ and Fengbing Luo ${ }^{3, c}$ \\ 1 University of Chinese Academy of Sciences, China \\ 2 SIASUN Robot and Automation Company Limited, China \\ ${ }^{3}$ SIASUN Robot and Automation Company Limited, China \\ afanzhen@sia.cn, bzhanglei@siasun.com, cluofengbing@siasun.com
}

Keyword: Wide-angle Lens; Center Iteration Method; Regional Interpolation Method

\begin{abstract}
In order to calibrate a wide-angle camera in real-time and find the precious position of the target object which could help the robot to find to location where they are, new method which uses the center iteration method and the regional interpolation method based on the distortion correction table is proposed. With this method, the world coordinates of the target object could be calculated accurately. The experimental results show that the theory is effective enough to get the world coordinates of objects with good accuracy for industrial application.
\end{abstract}

\section{Introduction}

With the developing of image processing technology and camera manufacturing technology, machine vision applications in the use of robotics get more attentions. Owning to the inflexible of the robot mechanical structure in many industrial conditions, a wide-angle camera which can obtain a larger field will be used to get the position of the target object. Because of the distortion which is caused by the wide-angle lens, the accuracy of the positioning result of the target object will be impacted.

[1] has introduced a good way for camera calibration. [2] has proposed the lens distortion model. And [3] - [6] have provided many different ways to complete the camera calibration. But these algorithms of camera calibration are too complex for a robot system to realize. So, this paper will propose an available method which could get the accuracy world coordinates with the center iteration method and the regional interpolation method.

\section{Wide-angle camera model}

Wide-angle camera model can be divided into the pinhole camera model and the lens distortion model. Consideredthese two different models, the paper proposes a method that could get the actual coordinate of target object is divided into two steps: First, with the lens distortion model, the distortion of the pixel coordinate will be eliminated. And second, the world coordinates will be calculated with the pinhole camera model.

A Pinhole camera model

With the pinhole model, the relationship between the pixel coordinates and the world coordinates could be obtained as $\left\{\begin{array}{l}u=\frac{f}{d u} \frac{X}{Z}+u 0 \\ v=\frac{f}{d v} \frac{Y}{Z}+v 0\end{array}\right.$, where $\mathrm{f}$ is the focal length of the camera, $\mathrm{du} \mathrm{dv}$ are the length of 
a pixel in the direction of $\mathrm{u} v$. Two variables, $\mathrm{Ku}=\mathrm{f} / \mathrm{du}$ and $\mathrm{Kv}=\mathrm{f} / \mathrm{dv}$, will be introduced. And $\mathrm{Ku}$ $\mathrm{Kv} \mathrm{u} 0 \mathrm{v} 0$ are intrinsic parameters of a certain camera and they would not be changed after a camera is produced completely. So, the matrix form of the pinhole model can be described as (1).

$$
\left(\begin{array}{c}
u t \\
v t \\
t
\end{array}\right)=\left(\begin{array}{ccc}
K u & 0 & u 0 \\
0 & K v & v 0 \\
0 & 0 & 1
\end{array}\right)\left(\begin{array}{c}
X \\
Y \\
Z
\end{array}\right)
$$

\section{B Lens distortion model}

There are mainly two main lens distortions in this model, radial distortion and tangential distortion. $(\mathrm{u}, \mathrm{v})$ is the ideal pixel coordinates in image plane without distortion, $\left(\mathrm{u}^{\mathrm{d}}, \mathrm{v}^{\mathrm{d}}\right)$ is the pixel coordinates with distortion. Similarly, (x, y) are the ideal image coordinates without distortion in image plant and $\left(\mathrm{x}^{\mathrm{d}}, \mathrm{y}^{\mathrm{d}}\right)$ is the distortional image coordinates. The distortion camera model [2] can be described by formula (2):

$$
\left\{\begin{array}{l}
u^{\mathrm{d}}=u+T_{\mathrm{u}}(\mathrm{r})+B_{\mathrm{u}}(\mathrm{r}) \\
v^{d}=v+T_{v}(r)+B_{\mathrm{v}}(\mathrm{r})
\end{array}\right.
$$

Where $\left\{\begin{array}{l}T_{\mathrm{u}}(\mathrm{r})=\left(\mathrm{u}-\mathrm{u}_{0}\right)\left(\mathrm{k}_{1} \mathrm{r}^{2}+\mathrm{k}_{2} \mathrm{r}^{4}+\mathrm{k}_{3} \mathrm{r}^{6}\right) \\ T_{\mathrm{v}}(\mathrm{r})=\left(\mathrm{v}-\mathrm{v}_{0}\right)\left(\mathrm{k}_{1} \mathrm{r}^{2}+\mathrm{k}_{2} \mathrm{r}^{4}+\mathrm{k}_{3} \mathrm{r}^{6}\right)\end{array},\left\{\begin{array}{l}B_{\mathrm{u}}(\mathrm{r})=2 p_{1} x_{d} y_{d}+p_{2}\left(r^{2}+2 x_{d}^{2}\right) \\ B_{\mathrm{v}}(\mathrm{r})=p_{1}\left(r^{2}+2 y_{d}{ }^{2}\right)+2 p_{2} x_{d} y_{d}\end{array}\right.\right.$. Equation (2) can be written in matrix form is shown as (3)

$$
A J=\mathrm{P}^{\mathrm{d}}-\mathrm{P}
$$

$$
\text { Where } P^{\mathrm{d}}=\left[u^{d}, \mathrm{v}^{d}\right]^{T}, P=[u, \mathrm{v}]^{T}, A=\left[\begin{array}{lllll}
\frac{x_{d}}{d u} r^{2} & \frac{x_{d}}{d u} r^{4} & \frac{x_{d}}{d u} r^{6} & 2 x_{d} y_{d} & \mathrm{r}^{2}+2 \mathrm{x}_{d}^{2} \\
\frac{y_{d}}{d u} r^{2} & \frac{y_{d}}{d u} r^{4} & \frac{y_{d}}{d u} r^{6} & \mathrm{r}^{2}+2 \mathrm{y}_{d}^{2} & 2 x_{d} y_{d}
\end{array}\right], J=\left[\mathrm{k}_{1}, \mathrm{k}_{2}, \mathrm{k}_{3}, \mathrm{p}_{1}, \mathrm{p}_{2}\right]^{T}
$$

$r^{2}=x^{2}+y^{2}, k_{1}, k_{2}, k_{3}$ are radial distortion coefficients, $p_{1}, p_{2}$ are the tangential distortion coefficients.

\section{Positioning method}

To obtain the world coordinates of target object, the positioning method is divided into four steps. First, calculate the distortion correspondence table. Secondly, obtain the pixel coordinates of the target object in the picture with distortion. Thirdly, eliminate the distortion of the pixel coordinates. And lastly, calculate the world coordinates of the target object with the pinhole camera model.

\section{A Distortion correspondence table}

With (3), the non-distortional pixel coordinates will be converted to the coordinates with distortion and then, the distortion correspondence tables could be obtained. Two double-precision floating point buffer, used to save the contents of the corresponding table, will be defined in this paper which are named $\mathrm{Fu}$ and Fv. With the following equation, one of the distortion correspondence tables could be obtained and stored in $\mathrm{Fu} . \mathrm{W}$ and $\mathrm{H}$ are defined as the width and height of the image size. 


$$
\left[\begin{array}{cccc}
F_{u}^{0,0} & F_{u}^{0,1} & \cdots & F_{u}^{0, \mathrm{~W}} \\
\vdots & \vdots & \vdots & \vdots \\
\cdots & \cdots & F_{u}^{\mathrm{i}, \mathrm{j}} & \cdots \\
\vdots & \vdots & \vdots & \vdots \\
F_{u}^{H-1,0} & F_{u}^{\mathrm{H}-1,1} & \cdots & F_{u}^{\mathrm{H}-1, \mathrm{~W}} \\
F_{u}^{\mathrm{H}, 0} & F_{u}^{\mathrm{H}, 1} & \cdots & F_{u}^{\mathrm{H}, \mathrm{W}}
\end{array}\right]=\left[\begin{array}{cccc}
0+A^{0,0} J & 0+A^{0,1} J & \cdots & 0+A^{0, \mathrm{~W}} J \\
\vdots & \vdots & \vdots & \vdots \\
\cdots & \cdots & \mathrm{i}+A^{\mathrm{i}, \mathrm{j}} J & \cdots \\
\vdots & \vdots & \vdots & \vdots \\
\mathrm{H}-1+A^{\mathrm{H}-1,0} J & \mathrm{H}-1+A^{\mathrm{H}-1,1} J & \cdots & \mathrm{H}-1+A^{\mathrm{H}-1, \mathrm{~W}} J \\
\mathrm{H}+A^{\mathrm{H}, 0} J & \mathrm{H}+A^{\mathrm{H}, 1} J & \cdots & \mathrm{H}+A^{\mathrm{H}, \mathrm{W}} J
\end{array}\right]
$$

B Pixel coordinates correction

This paper introduces the center iteration method and the regional interpolation method to get the non-distortion pixel coordinates. The center iteration method will be used to obtain the minimum range of the non-distortion pixel coordinates of the target object minimum range, and the sub-pixel coordinates will be gotten with the regional interpolation.

The minimum range of the non-distortion pixel coordinates will be found with the center iteration method. Initializing the searching range of $(\mathrm{u}, \mathrm{v})$ should be finished as first. And then ascertaining the quadrant of the target object after the comparing the pixel coordinates of the center point to the pixel coordinates of target object. Thirdly, resetting the searching range of $(\mathrm{u}, \mathrm{v})$ according to the results of 2 . Lastly, determining whether the result could meet the requirements of the vision robot system or not.

The pixel coordinates of the target object with distortion which are obtained by the image process are integer. Nearest Neighbor Interpolation can't ensure the accuracy of final pixel coordinates with the minimum range which is defined in the Center iteration method. So, the regional interpolation method is proposed by this paper to make sure the accuracy results. With this method, an accurate pixel coordinates without distortion can be calculated and it will be used to get the world coordinates. $\left[\mathrm{M}_{1}, \mathrm{M}_{2}\right],\left[\mathrm{N}_{1}, \mathrm{~N}_{2}\right]$ are defined as the minimum searching range of $\mathrm{u}$ and $\mathrm{v}$. So, the description of the regional interpolation method is shown in (4).

$$
\left\{\begin{array}{l}
\mathrm{u}=\sum_{i=M_{1}}^{M_{2}} \sum_{j=\mathrm{N}_{1}}^{N_{2}}[g(\mathrm{i}, \mathrm{j}) \times \mathrm{Fu}(\mathrm{i}, \mathrm{j})] \\
\mathrm{v}=\sum_{i=M_{1}}^{M_{2}} \sum_{j=\mathrm{N}_{1}}^{N_{2}}[g(\mathrm{i}, \mathrm{j}) \times \mathrm{Fv}(\mathrm{i}, \mathrm{j})]
\end{array}\right.
$$

Where $g(\mathrm{i}, \mathrm{j})=\frac{\frac{1}{\sqrt{(\mathrm{i}-\mathrm{u})^{2}+(\mathrm{j}-\mathrm{v})^{2}}}}{\sum_{p=M_{1}}^{M_{2}} \sum_{q=\mathrm{N}_{1}}^{N_{2}} \frac{1}{\sqrt{(\mathrm{p}-\mathrm{i})^{2}+(\mathrm{q}-\mathrm{j})^{2}}}}$ represents the weight value of the adjacent pixel coordinates which located in the row $\mathrm{i}$ and column $\mathrm{j}$.

The distortions of the pixel coordinates will be eliminated through the center iteration method and the regional interpolation method. In order to verify the feasibility of the method, an experiment is designed with a grid image which is taken by a camera with wide-angle lens as input image.by collecting. The experimental results are shown in Fig. 1. The results show that the method can be used to correct the distortion caused by the wide-angle lens. The center part receives the best effect of the distortion correcting and some distortions in the edge part of the image which is still meeting the requirements. 


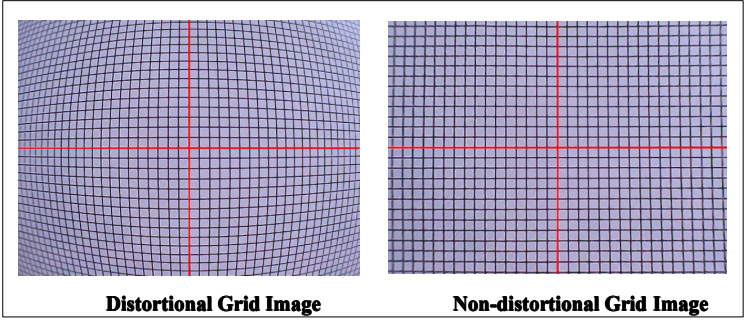

Figure 1 Anti-distortion Experiment Result

\section{Experiment}

In order to verify the accuracy of the actual coordinates which is calculated by the method proposed in this paper, an experiment is designed with the AGV which is produced by SIASUN Co. Ltd. A camera with wide-angle lens is installed in the chassis of the vehicle and will be used to identify the target object. The data shown in Table 1 has record the world coordinates calculated by the method which is proposed in this paper and the coordinates which is measured in real.

The way that is chosen in this paper to estimate the accuracy of the positioning method is ARE (Averaged Residual Error), and (Maximum Residual Error) [6], which are defined in formula (5):

$$
\left\{\begin{array}{c}
A R E=\frac{1}{\mathrm{n}} \sum_{i=1}^{n} \sqrt{\left(\mathrm{X}_{i}^{\text {real }}-\mathrm{X}_{i}\right)^{2}+\left(\mathrm{Y}_{i}^{\text {real }}-\mathrm{Y}_{i}\right)^{2}} \\
M R E=\max \left\{\sqrt{\left(\mathrm{X}_{i}^{\text {real }}-\mathrm{X}_{i}\right)^{2}+\left(\mathrm{Y}_{i}^{\text {real }}-\mathrm{Y}_{i}\right)^{2}}\right\}
\end{array}\right.
$$

Where $\left(\mathrm{X}_{i}, \mathrm{Y}_{i}\right)$ are the coordinates calculated by the center iteration method and the regional interpolation method. $\left(\mathrm{X}_{\mathrm{i}}^{\text {real }}, \mathrm{Y}_{i}^{\text {real }}\right)$ are the coordinates which are measured in real. The experimental data is recorded in Tab. 1. From the experimental data, the assessments are $\mathrm{ARE}=0.35, \mathrm{MRE}=0.89$

ARE shows that the average error of the world coordinates calculated by the method is less than $0.5 \mathrm{~mm}$. The maximum measurement error shown by the MRE also can meet the requirement for the accuracy. Therefore, the results show that the method proposed by this paper can achieve a high accuracy positioning result of the target object.

The CPU in this experiment is OMAP3530 produced by TI with a clock speed of $600 \mathrm{MHz}$. In order to verify the real-time of this algorithm, experiments measured the time consuming of the process of calculating the pixel coordinates without distortion with the center iteration method and the regional interpolation method. With the experiment of the real-time evaluation, the average time consuming of correcting the distortion is $0.35 \mathrm{~ms}$. As the control cycle of the vehicle is about $50 \mathrm{~ms}$, the anti-distortion process wouldn't cause great time consumption and it can meet the real-time requirements of AGV.

\section{Summary}

The wide-angle camera will be used sometimes in the visual positioning system to get the target object in the large field of view. The problem which is difficult to get the accuracy position of target object with the distortion of wide-angle lens is becoming prominent. To eliminate the distortion, this paper proposed the center iteration method and the regional interpolation method to get the pixel coordinates without distortion and with the pinhole camera model, the actual coordinates of the target object would be gotten. By using the center iteration method, the pixel coordinates range which is without distortion could be obtained in real-time. At the same time, the regional interpolation method will ensure the accuracy of the positioning. The experimental results also proved that the accuracy could meet the requirements. 
Table 1 Experimental Data of Coordinates

unit: $\mathrm{mm}$

\begin{tabular}{|c|c|c|c|c|c|}
\hline \multirow{2}{*}{ No. } & \multicolumn{2}{|c|}{ Calculated coordinates } & \multicolumn{2}{c|}{ Real coordinates } & \multirow{2}{*}{ Residual error } \\
\cline { 2 - 5 } & $\mathrm{X}$ & $\mathrm{Y}$ & \multicolumn{2}{c|}{$\mathrm{X}^{\text {real }}$} & \multicolumn{2}{c|}{$\mathrm{Y}^{\text {real }}$} & \\
\hline 1 & -4.1 & 3.1 & -4 & 2.3 & 0.81 \\
\hline 2 & 14.2 & -15.2 & 14 & 15.5 & 0.36 \\
\hline 3 & -10.2 & -2.5 & -10 & -3 & 0.54 \\
\hline 4 & -18.9 & -16.7 & -18.30 & -16.5 & 0.63 \\
\hline 5 & 18.6 & -13.91 & 18 & 13.5 & 0.73 \\
\hline 6 & -5.7 & 1.31 & -5.5 & 1.2 & 0.23 \\
\hline 7 & -1 & -0.6 & -1 & -0.5 & 0.1 \\
\hline 8 & 3.1 & -3.1 & 3 & -3 & 0.14 \\
\hline 9 & 9.4 & 8.8 & 9.8 & 8 & 0.89 \\
\hline 10 & 13.8 & 7.7 & 13.7 & 7.8 & 0.14 \\
\hline 11 & 11.2 & 12.6 & 11 & 12.8 & 0.28 \\
\hline 12 & 0.4 & -0.06 & 0.1 & 0 & 0.31 \\
\hline 13 & 3.7 & -0.06 & 3.5 & 0 & 0.21 \\
\hline 14 & -0.1 & 4.4 & -0.1 & 4.2 & 0.2 \\
\hline 15 & 2.15 & 7.6 & 2 & 7.8 & 0.25 \\
\hline 16 & -15.4 & -7.4 & -15 & 7.8 & 0.41 \\
\hline 17 & 3.3 & 7.75 & 3.5 & 7.9 & 0.25 \\
\hline 18 & 5.81 & 2.3 & 5.9 & 2.3 & 0.09 \\
\hline 19 & -0.7 & 1.9 & -0.8 & 2 & 0.14 \\
\hline 20 & 2.2 & -1.7 & 2 & -1.8 & 0.22 \\
\hline
\end{tabular}

\section{References}

[1]Zhengyou Zhang. A Flexible New Technique Technique for Camera Calibration [J]. IEEE T ransactions on Patter Analysis and Machine Intelligence,2000, 22(11) for Camera Calibration [J]. IEEE Transactions on Patter Analysis and Machine Intelligence, 2000, 22 (11)

[2]Duane C. Brown. Close-Range Camera Calibration [J]. Photogrammetric Engineering. 1971.

[3]Tsai R YA versatile camera calibration technique for high accuracy 3D machine vision metrology using off-the-Shelf TV camera and lenses [J]. IEEE Journal of Robotics and Automation, 1987 3 (4): 323-344

[4]Fu Dan, Zhou Jian, Qiu Zhiqiang, etc. Based on geometric invariant linear camera calibration parameters [J]. Chinese Journal of Image and Graphics, 2009,14 (6): 1058-1063

[5]Wang Zhiliang, Zhang Qiong, CHI Jian-nan, etc. Based on the straight-line projection of the camera parameter calibration feature [J]. Computer Science, 2011, 38 (8): 272-274.

[6]C. Brauer-Burchardt . Brauer-Burchardt. A simple new method for precise lensdistortion correction of low cost camera systems $[\mathrm{J}]$. A simple new method for precise lens distortion correction of low cost camera systems [J]. DAGM 2004 . 3175 . 570-577 DAGM 2004. 3175. 570-577

[7]Gary Bradski, Adrian Kaeler. Learning OpenCV [M]

[8]The Distortion Correction of Large View Wide-angle Lens for Image Mosaic Based on OpenCV [J]. International Conference on Mechatronic Science, Electric Engineering and Computer [C], China, August $19-22,2011,1074-1077$ 